\title{
MELHORIA NO PROCESSO DE GESTÃO DE ESTOQUE EM UMA CONFECÇÃO DO RAMO TÊXTIL: UM ESTUDO DE CASO
}

\section{IMPROVEMENT IN THE INVENTORY MANAGEMENT PROCESS IN A CONFECTION OF THE TEXTILE BRANCH: A CASE STUDY}
Emmanuel Belga Rodrigues ${ }^{1}$, Maria Eduarda Belga Rodrigues ${ }^{2 *}$ Mário César Fialho de ${\underline{\text { Oliveira }^{3}}}^{3} \underline{\text { Sanderson Rocha de Abreu }}^{4}, \&$ Antônio Elízio de Oliveira $^{5}$
12345 Faculdades Integradas de Catagueses.
emmanuel.rodrigues@alunos.uni.edu.br ${ }^{2 *}$ dudabelga@gmail.com ${ }^{3}$ mc-mariocesar@hotmail.com
${ }^{4}$ sanderson.abreu@alunos.unis.edu.br ${ }^{5}$ antonio.oliveira@ professor.unis.edu.br

\section{ARTIGO INFO.}

Recebido em: 01.10.2021

Aprovado em: 25.10.2021

Disponibilizado em: 16.11.2021

\section{Palavras-chave:}

Sistema de Revisão Contínua; Sistema de Revisão Periódica; Indicadores de Desempenho de estoque; Gestão de Estoque.

\section{KEYWORDS:}

Continuous Review System; Periodic Review System; Inventory Performance Indicators; Inventory Management.

\footnotetext{
*Autor Correspondente: Rodrigues, M. E. B.

RESUMO

O estudo e a concepção de modelos de gestão de estoque, baseados nas metodologias de revisão contínua e revisão periódica, permitem medir a maneira mais eficiente de controle de estoque, avaliando de forma quantitativa os efeitos desses modelos na performance do estoque. Assim sendo, através do ambiente de simulação dos cálculos propostos, o presente estudo busca determinar o método ideal para a política de ressuprimento de uma empresa do ramo têxtil. Os modelos de controle de estoque são comparados através de indicadores, desempenhando uma redução nos custos totais do estoque, onde um deles é escolhido como o mais viável para a empresa estudada.
}

\begin{abstract}
The study and design of inventory management models, based on continuous review and periodic review methodologies, allows us to evaluate the most efficient way to control stock, evaluating the quantitative way of the effects of these models on stock performance. Therefore, through the simulation environment of the proposed calculations, this study seeks to determine the ideal method for the resupply policy of a textile company. The inventory control models are compared through indicators, reducing total inventory costs, where one of them is selected as the most viable for the studied company.
\end{abstract}


Citação (APA): Rodrigues, E. B., Rodrigues, M. E. B., Oliveira, M. C. F., de, Abreu, S. R., de, \& Oliveira, A. E., de. (2021). Melhoria no processo de gestão de estoque em uma confecção do ramo têxtil: Um estudo de caso. Brazilian Journal of Production Engineering, 7(5), 8292.

\section{INTRODUÇÃO}

A gestão de estoque pode apresentar grandes problemas no processo de produção, afetando, muitas vezes, a entrega e a qualidade do produto. De acordo com Slack (2002), manter itens estocados representa riscos, mas também é uma garantia para incertezas. O controle dos itens no estoque da confecção estudada é realizado de forma manual, onde um funcionário realiza a contagem dos itens estocados na entrada e saída dos mesmos. A gestão de estoque implementada é a mesma utilizada desde a inserção da empresa no mercado.

A principal função ou objetivo da gestão de estoque consiste na busca para achar o equilíbrio entre estoque e consumo, visto isto, a necessidade de um método de controle de estoques é de extrema importância para o melhor funcionamento da empresa. Uma eficiente gestão de estoques possibilita à organização obter melhorias significativas na sua administração, uma vez que repercute em uma melhora na eficiência da realização da produção planejada, traz maior segurança nas tomadas de decisões, além de prevenir possíveis atrasos na entrega dos pedidos.

Portanto, a proposta de um sistema para controle de materiais vindo a ser implementado, servirá para um maior controle de todos os itens que a empresa possui, visto que se há acúmulo de materiais, gera um custo muito elevado e em contrapartida a falta de um estoque também acarreta em altos custos financeiros. O controle deste, facilitará as atividades da empresa, pois pode-se ter mais segurança, agilidade e controle, na hora de vender e produzir um produto, visto que, com o controle de ativos não precisa preocupar-se que faltará algum item para a produção.

O objetivo geral deste estudo é a estruturação de uma proposta para o sistema de gestão no controle de estoque, em uma empresa no segmento têxtil na cidade de Cataguases - Minas Gerais. Identificando o modelo de gestão ideal para o controle de entradas e saídas.

\section{REVISÃO DE LITERATURA}

\subsection{Gestão de Estoque}

O estoque é por definição, de acordo com Paoleschi (2014), qualquer quantidade de bens físicos que sejam conservados, de forma improdutiva, em um intervalo de tempo. Gasnier (2002) elucida a definição de estoque afirmando que, configura-se em estoque, qualquer produto que não está em movimento, ou seja, não estão em fluxo, onde ficam em depósitos, almoxarifados, pátios ou armazéns.

Entende-se como gestão de estoque o planejamento do estoque, seu controle e sua retroalimentação de acordo com o planejamento. Consistindo em planejar o estoque, determinar a volumetria de materiais que entram e saem, bem como as datas em que ocorrem suas entradas e saídas, o tempo que decorrem entre essas datas e os pontos de pedidos (CHING, 2010).

De acordo com Martins e Alt (2009) a gestão de estoques constitui uma série de ações que permitem ao administrador verificar se os estoques estão sendo bem utilizados, bem localizados em relação aos setores que deles se utilizam, bem manuseados e bem controlados.

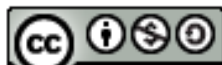


Citação (APA): Rodrigues, E. B., Rodrigues, M. E. B., Oliveira, M. C. F., de, Abreu, S. R., de, \& Oliveira, A. E., de. (2021). Melhoria no processo de gestão de estoque em uma confecção do ramo têxtil: Um estudo de caso. Brazilian Journal of Production Engineering, 7(5), 8292.

\subsection{Método de Ressuprimento}

Peinado e Graeml (2007) ressalta que para definir o método de ressuprimento, primeiro se faz necessário estabelecer a forma como os estoques são constantemente verificados e reabastecidos, portanto, consiste em definir quando será reabastecido e a quantidade que será reposto.

\subsubsection{Sistema de Revisão Contínua}

O modelo de revisão contínua estabelece que os processos de controle de estoque devem ser realizados diariamente, com a finalidade de verificar a necessidade de suprir o estoque. $\mathrm{O}$ procedimento de ressuprimento inicia-se quando o estoque atinge um nível estabelecido pelo ponto de pedido (PP) ou ponto de ressuprimento (PR). (Bowersox \& Closs, 2011).

Para Wank (2011), o valor k para o cálculo de estoque de segurança representa o nível de certificação para que não ocorra a falta do produto para um determinado nível de serviço. Através do valor do nível de serviço é possível encontrar o valor de k, O fator de segurança pode ser identificado derivado aos vários níveis de serviços, conforme Corrêa e Corrêa (2012). Respectivamente, o fator de serviço 1,$037 ; 1,282 ; 1,645 ; 1,751 ; 1,880 ; 2,055 ; 2,325$ e 3,100 são definidos pelo nível de serviço desejado de 85\%; 90\%; 95\%; 96\%; 97\%; 98\%; 99\% e 99,9\%..

Tabela 1. Fórmulas do Sistema de Revisão Contínua

\begin{tabular}{llll}
\multicolumn{1}{c}{ Nome } & \multicolumn{1}{c}{ Descrição } & Mensuração & \multicolumn{1}{c}{ Literatura } \\
\hline Ponto do pedido & $\begin{array}{l}\text { Ponto determinado para } \\
\text { ressuprimento. }\end{array}$ & PP=D*TR+ES & Bowersox e Closs, 2011 \\
\hline Estoque de Segurança & $\begin{array}{l}\text { Mantem níveis de estoque } \\
\text { suficientes para evitar faltas }\end{array}$ & ES=k* $\sigma^{*} \sqrt{L} T$ & Martins e Campos (2009) \\
\hline Lote econômico de compra & $\begin{array}{l}\text { Determina a quantidade de } \\
\text { compra minimizando os } \\
\end{array}$ & LEC $=\sqrt{ }\left(2^{*} \mathrm{CP} \mathrm{D}^{*} / \mathrm{i}^{*} \mathrm{P}\right)$ & Slack et al. (2018) \\
& & & \\
\hline
\end{tabular}

Fonte: Autores (2021).

\subsubsection{Sistema de Revisão Periódica}

Neste modelo de revisão periódica, Chopra e Chopra e Meindl (2016) destaca que níveis de estoques são verificados em conformidade com um período de tempo estipulado em dias, semanas ou meses. Distinguindo do modelo de revisão continua, o tamanho dos pedidos pode variar, seguindo a demanda expressada entre os pedidos sucessivos e o estoque conseguinte no momento do pedido.

Tabela 2. Fórmulas do Sistema de Revisão Periódica

\begin{tabular}{|c|c|c|c|}
\hline Nome & Descrição & Mensuração & Literatura \\
\hline Tempo ótimo entre as revisões & $\begin{array}{l}\text { Tempo entre as revisões do } \\
\text { nível do estoque. }\end{array}$ & $\operatorname{Tr}=\operatorname{Tano} /\left(\mathrm{N}^{*}\right)=\left(\mathrm{Q}^{*} \times \operatorname{Tano}\right) / \mathrm{D}$ & Tubino (2000) \\
\hline Estoque de Segurança & $\begin{array}{l}\text { Mantem níveis de estoque } \\
\text { suficientes para evitar faltas. }\end{array}$ & $E S=k^{*} \sigma^{*} V(I P+L T)$ & Martins e Campos (2009) \\
\hline Estoque máximo & $\begin{array}{l}\text { Parêmetro para determinar o } \\
\text { nível máximo do estoque. }\end{array}$ & Emáx $=E S+Q$ & Dias (2010) \\
\hline Quantidade de novo pedido & $\begin{array}{l}\text { Quantidade de material } \\
\text { necessário para } \\
\text { ressuprimento. }\end{array}$ & Qn=Emáx-E-Qp & Bowesorx e Closs (2011) \\
\hline Lote econômico de compra & $\begin{array}{l}\text { Determina a quantidade de } \\
\text { compra minimizando os } \\
\text { custos. }\end{array}$ & $\mathrm{LEC}=\sqrt{ }\left(2^{*} \mathrm{CP} \mathrm{P}^{*} \mathrm{D} / \mathrm{i}^{*} \mathrm{P}\right)$ & Slack et al. (2018) \\
\hline
\end{tabular}

Fonte: Autores (2021). 
Citação (APA): Rodrigues, E. B., Rodrigues, M. E. B., Oliveira, M. C. F., de, Abreu, S. R., de, \& Oliveira, A. E., de. (2021). Melhoria no processo de gestão de estoque em uma confecção do ramo têxtil: Um estudo de caso. Brazilian Journal of Production Engineering, 7(5), 8292.

\subsection{Indicadores}

A Tabela 3 discrimina os indicadores que auxiliam na medição do desempenho de estoque, sendo eles: Estoque Médio, Giro de Estoque, Cobertura de Estoque, Custo do pedido, Custo da manutenção, Custo da falta e Custo total do estoque.

Tabela 3. Fórmulas dos Indicadores

\begin{tabular}{|c|c|c|c|}
\hline Nome & Descrição & Mensuração & Literatura \\
\hline Estoque médio & $\begin{array}{l}\text { Nível médio dos itens em estoque } \\
\text { no período determinado }\end{array}$ & $\mathrm{EM}=($ Estoque inicial+Estoque final $) / 2$ & Dias (2010) \\
\hline Giro de Estoque & $\begin{array}{l}\text { Indica o volume de estoque que a } \\
\text { empresa irá precisar ter no início } \\
\text { do mês para cobrir a demanda } \\
\text { prevista para o período. }\end{array}$ & $\begin{array}{l}\text { Giro de estoque }=(\text { Venda total no } \\
\text { período }) /(\text { Estoque médio do período })\end{array}$ & Parente (2009) \\
\hline $\begin{array}{l}\text { Cobertura de } \\
\text { Estoque }\end{array}$ & $\begin{array}{l}\text { Determina a quantidade em tempo } \\
\text { em que o estoque foi suficiente } \\
\text { para atender a demanda. }\end{array}$ & $\begin{array}{l}\text { Corbetura de estoque }=(\text { Período de } \\
\text { tempo analisado)/(Giro de estoque ) }\end{array}$ & $\begin{array}{l}\text { Moura e Silva } \\
\text { (2012) }\end{array}$ \\
\hline Custo do pedido & $\begin{array}{l}\text { Todos os custos usados para } \\
\text { reabastecer o estoque e que } \\
\text { configuram os custos para a } \\
\text { empresa. }\end{array}$ & $\mathrm{CP}=(\mathrm{CG} * \mathrm{TG})+(\mathrm{CS} * \mathrm{TS})+(\mathrm{CL} * \mathrm{TL})$ & Slack et al. (2018) \\
\hline $\begin{array}{l}\text { Custo da } \\
\text { manutenção }\end{array}$ & $\begin{array}{l}\text { Custo de se manter um material } \\
\text { em estoque por um intervalor de } \\
\text { tempo não estabelecido }\end{array}$ & $\mathrm{CE}=\mathrm{t}^{*} \mathrm{Cu} * \mathrm{LC} / 2$ & Moreira (2012) \\
\hline Custo da falta & $\begin{array}{l}\text { Custo da falha da decisão do } \\
\text { pedido pela sua quantidade o que } \\
\text { ocasiona a falta do material no } \\
\text { estoque }\end{array}$ & $\mathrm{CF}=\mathrm{PC} * \mathrm{TLL}{ }^{*} \mathrm{P}$ & Slack et al. (2018) \\
\hline Custo total & $\begin{array}{l}\text { Indica o custo total gerado pelo } \\
\text { estoque }\end{array}$ & $\mathrm{CT}=\left((\mathrm{NP} * \mathrm{CP})+\left(\mathrm{QF} \mathrm{F}^{*} \mathrm{CF}\right)+\left(\mathrm{EM} \mathrm{M}^{*} \mathrm{CE}\right)\right)$ & Tubino (2007) \\
\hline
\end{tabular}

Fonte: Autores (2021).

\section{METODOLOGIA}

Para elaboração do presente trabalho foram realizadas pesquisas bibliográficas em artigos científicos, revistas e sites acadêmicos, no período de fevereiro de 2021 a junho de 2021. A metodologia utilizada foi um estudo de caso, de modo que as pesquisas realizadas devam se associar a teoria e a ação, com a finalidade que a situação ou o problema seja resolvido. Atualmente a empresa estudada não possui metodologia de gestão de estoque e o processo de ressuprimento não segue um método de compra para atender a demanda, baseando-se no conhecimento empírico na experiência do responsável. De acordo com Pozo (2010) é praticamente impossível prever com exatidão a demanda futura, sendo necessário definir um nível de estoque para garantir a disponibilidade de produtos. Deste modo, é preciso uma metodologia adequada para que não se tenha excesso ou ruptura de material.

3.1. Selecionar as principais matérias-primas, através da curva ABC.

Os dados foram coletados por meio do sistema ERP utilizado pela empresa e foi estruturado em uma planilha da ferramenta Excel ${ }^{\circledR}$, foi utilizado um relatório de entradas e saídas do estoque no período de 01/01/2021 a 30/06/2021. 

processo de gestão de estoque em uma confecção do ramo têxtil: Um estudo de caso. Brazilian Journal of Production Engineering, 7(5), 8292.

Na confecção estudada, as matérias-primas são compostas por três famílias que são: malha, tecido e aviamentos. Suas unidades de medidas podem ser em: quilo (kg), metro (m) e unidade (um).

Segundo Soto et al. (2017), a análise da Curva ABC consiste em dividir os itens de uma determinada empresa em três grupos com base na lucratividade que é gerada para a empresa. $\mathrm{O}$ Grupo A é o grupo de itens mais valiosos, e representam $80 \%$ do custo total anual, mas apenas $20 \%$ do total de itens em estoque. O Grupo B consiste em ser $15 \%$ do custo total, representando $30 \%$ dos itens estocados. E, o Grupo C, possui os itens com menor custo, em 5\% do total, onde representa a maior parte de itens em estoque, totalizando $50 \%$.

Por se tratar de três famílias de matérias-primas, com diferentes valores de custo e consumo, foi elaborada uma classificação ABC para as famílias, utilizando o custo total por família sobre o custo geral do estoque, para determinar qual família de matéria prima é a mais relevante financeiramente no estoque.

Figura 1. Gráfico Curva ABC - Famílias de Matérias-primas

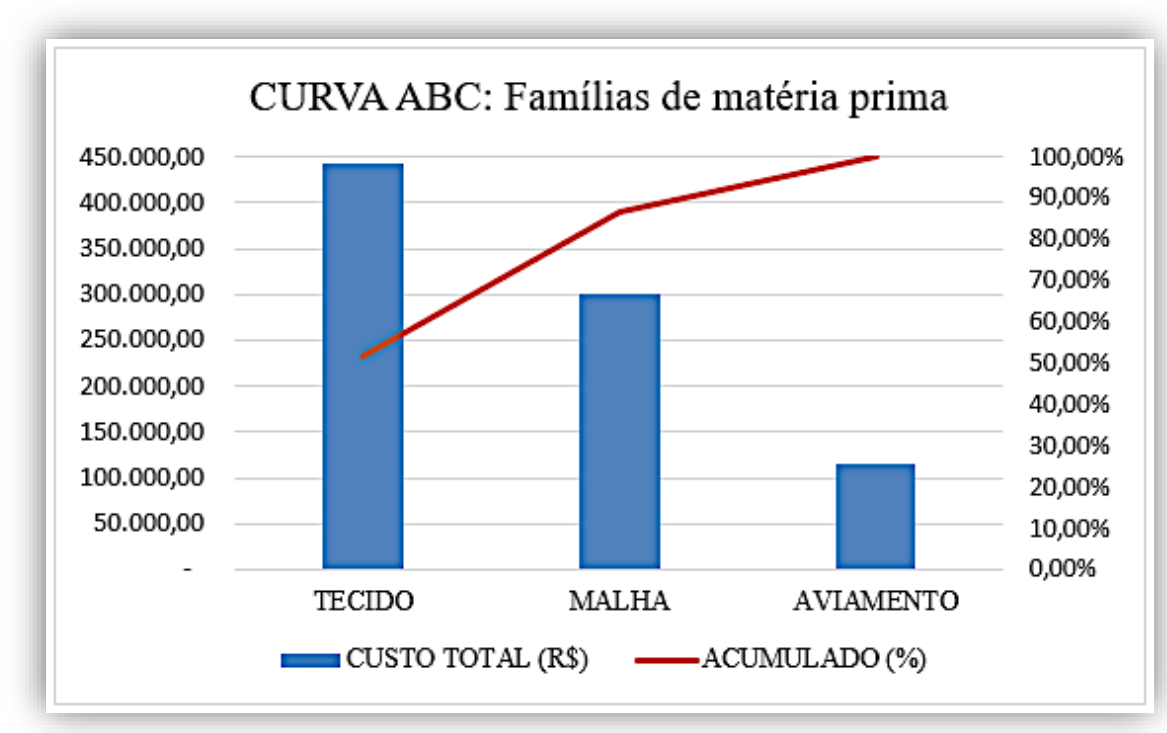

Fonte: Autores (2021).

Observa-se que, a partir da curva $\mathrm{ABC}$ por famílias de matérias-primas, a família de Tecido representa a classe A com 51,58\%. A família de Malhas representa a classe B com 34,97\% e, por fim, a família de Aviamentos representa a classe C com 13,45\%. Desta maneira, foi selecionada a família de Tecidos para determinar o item de maior relevância financeiramente no estoque.

Tabela 4. Classificação ABC de matéria-prima - Família de Tecidos

\begin{tabular}{lcrrrrc} 
Descrição & GRUPO & VALOR & PARTICIPAÇÃO ACUMULADO CLASSE \\
\hline MICROFIBRA CALIFORNIA PESADA & TECIDO & 35427,31 & 0,079827371 & 0,079827371 & A \\
\hline & $\vdots$ & & & & & \\
& $\vdots$ & & & & & \\
TE01190100100000164827001D02 CAPITU CALANDRADO 1,4 & TECIDO & 86,16 & 0,000194142 & 0,999109011 & $\mathrm{C}$ \\
\hline TE01190100100000164612001D02 CAPITU CALANDRADO 1,4 & TECIDO & 71,8 & 0,000161785 & 0,999270796 & $\mathrm{C}$ \\
\hline 030 POLIESTER 100\% KG ESCURO FURADO 0013 PRETO & TECIDO & 60,3 & 0,000135872 & 0,999406668 & $\mathrm{C}$ \\
\hline
\end{tabular}

Fonte: Autores (2021). 
A escolha dos itens a serem utilizadas para análise neste estudo é ponderada através da classificação entre as famílias e sua representatividade, onde se selecionou o item de maior relevância da Família Tecidos, Microfibra California Pesada que retrata 7,98\%.

\subsection{Simulação do modelo atual}

A tabela 5 apresenta as seguintes informações: consumo mensal de janeiro de 2021 a junho de 2021; consumo médio que foi calculado através somatório dos valores de consumo mensal divido pelo tempo estudado, seis meses; e o desvio padrão do consumo, calculado através da formula "DESVPADA" disponível no Excel, que estima o desvio padrão de uma amostra, onde a amostra é o consumo de janeiro a junho de 2021

Tabela 5. Resumo de consumo do item Microfibra California Pesada

\begin{tabular}{lcccccc} 
Meses & jan/21 & fev/21 & mar/21 & abr/21 & mai/21 & jun/21 \\
\hline Consumo & 1738 & 0 & 234,96 & 661,76 & 1586,64 & 1606,88 \\
\hline Consumo Médio & \multicolumn{7}{c}{971,37} \\
\hline Desvio Padrão do Consumo & \multicolumn{7}{c}{768,36} \\
\hline
\end{tabular}

Fonte: Autores (2021).

\subsubsection{Resultados dos indicadores de desempenho no modelo atual}

Conforme a fórmula do estoque médio na tabela 3 , foi calculado o estoque médio mensal do período estudado, janeiro a junho de 2021, onde seguiu-se para o cálculo da média dos valores encontrados da média do estoque mensal.

Ainda de acordo com a tabela 3, considerou-se as fórmulas dos autores citados para o cálculo do giro de estoque e cobertura de estoque apontados na tabela 6.

Tabela 6. Modelo de gestão atual do item Microfibra California Pesada

\begin{tabular}{lcccccc} 
Meses & jan/21 & fev/21 & mar/21 & abr/21 & mai/21 & jun/21 \\
\hline Estoque médio & 3557,67 & 4291,70 & 4154,51 & 4149,76 & 4547,66 & 4881,09 \\
\hline Média do Estoque médio & \multicolumn{7}{c}{4263,73} \\
\hline$N^{0}$ de Pedidos & 1 & 0 & 0 & 1 & 1 & 0 \\
\hline$\Sigma$ Faltas & \multicolumn{7}{c}{0} \\
\hline Giro de Estoque & \multicolumn{7}{c}{$1,37,68$} \\
\hline Cobertura de Estoque & \multicolumn{7}{c}{131,68} \\
\hline
\end{tabular}

Fonte: Autores (2021).

Os resultados dos custos totais obtidos na tabela 7 foram calculados por meio da teoria descrita na tabela 3, submetendo-se ao custo de pedido; custo da manutenção, sendo que o valor da taxa de oportunidade foi obtido pela taxa de rendimento em aplicações cotado no primeiro semestre de 2021; e custo da falta, onde o produto estudado não é comercializado de forma integral, ou seja, não existe um preço de venda para este produto, deste modo foi utilizado o custo unitário para as análises e a taxa de lucro líquido foi fornecida pelo setor financeiro, já o peso, foi definido pelo gestor do estoque, tendo em vista que, o item estudado representa o insumo dos produtos que mais possuem volumetria de vendas. 
Citação (APA): Rodrigues, E. B., Rodrigues, M. E. B., Oliveira, M. C. F., de, Abreu, S. R., de, \& Oliveira, A. E., de. (2021). Melhoria no processo de gestão de estoque em uma confecção do ramo têxtil: Um estudo de caso. Brazilian Journal of Production Engineering, 7(5), 8292.

Tabela 7. Resultados do custo total

\begin{tabular}{|c|c|c|c|c|c|c|c|c|c|c|c|c|}
\hline Meses & \multicolumn{2}{|c|}{ jan/21 } & \multicolumn{2}{|c|}{ fev/21 } & \multicolumn{2}{|c|}{$\operatorname{man} / 21$} & \multicolumn{2}{|c|}{$a b r / 21$} & \multicolumn{2}{|c|}{$\mathrm{mai} / 21$} & \multicolumn{2}{|c|}{ jun/21 } \\
\hline Custo total de Pedido & RS & 4,85 & RS & - & RS & - & RS & 4,85 & RS & 4,85 & RS & - \\
\hline Núme ro dos pedidos (NP) & \multicolumn{2}{|c|}{1} & \multicolumn{2}{|c|}{0} & \multicolumn{2}{|c|}{0} & \multicolumn{2}{|c|}{1} & \multicolumn{2}{|c|}{1} & \multicolumn{2}{|c|}{0} \\
\hline Custo de Pedidos (CP) & RS & 4,85 & RS & 4,85 & RS & 4,85 & RS & 4,85 & RS & 4,85 & RS & 4,85 \\
\hline
\end{tabular}

\begin{tabular}{|c|c|c|c|c|c|c|}
\hline Custo total de Manutenção & RS $92.795,07$ & RS $111.940,86$ & RS $108.362,39$ & RS $108.238,50$ & RS $118.617,00$ & RS $127.313,99$ \\
\hline Estoque Médio (EM) & 3557,67 & $\mathbf{4 2 9 1 , 7 0}$ & 4154,51 & 4149,76 & 4547,66 & 4881,09 \\
\hline Custo de Estocagem (CE) & 26,08 & 26,08 & 26,08 & 26,08 & 26,08 & 26,08 \\
\hline
\end{tabular}

\begin{tabular}{|c|c|c|c|c|c|c|}
\hline Custo total de Falta & RS & RS & RS & RS & RS & RS \\
\hline Quantidade de Falta (QF) & 0 & 0 & 0 & 0 & 0 & 0 \\
\hline Custo de Falta (CF) & 3,67 & 3,67 & 3,67 & 3,67 & 3,67 & 3,67 \\
\hline
\end{tabular}

\begin{tabular}{|c|l|l|l|l|l|l|}
\hline Custo do Total do Estoque & RS 92.799,91 & RS 111.940,86 & RS 108.362,39 & RS $108.243,34$ & RS 118.621,85 & RS 127.313,99 \\
\hline$\Sigma$ Custos Totais Mensais & RS & & & $667.282,35$ \\
\hline
\end{tabular}

Fonte: Autores (2021).

\subsection{Modelo de revisão contínua}

A metodologia do modelo de revisão continua tem como princípio a verificação constante do nível do estoque visando atender a necessidade de ressuprimento, desta maneira é determinado o ponto de pedido (PP) e quando o nível de estoque atinge o ponto de pedido ele é suprido pela quantidade determinada pelo lote econômico de compra (LEC).

Para obter o Ponto de Pedido (PP) é necessário o cálculo do estoque de segurança, onde conforme apresentado na tabela 1 é considerado os seguintes parâmetros: desvio padrão da demanda, já calculado na tabela 5; o lead time que para o fornecedor deste item é considerado 5 dias; o Fator de Segurança que conforme definido por Corrêa e Corrêa (2012) corresponde a 2,005, dado que o nível de serviço adotado foi de $98 \%$. Justificando essa escolha pelo fato de que a demanda por este produto é muito incerta e quanto maior o nível de serviço, maior o estoque médio, desta maneira optou-se por manter um estoque de segurança maior.

O Ponto de Pedido de acordo com Bowersox e Closs (2011), descrito na Tabela 1, obtém-se pelo consumo médio mensal, calculado na tabela 5; tempo de resposta médio que corresponde ao lead time; e o estoque de segurança.

O Lote Econômico de Compra é calculado através do Custo do Pedido; a Demanda Média; Taxa de Oportunidade; e o Preço Unitário do item estudado.

\subsection{Modelo de revisão periódica.}

A metodologia de revisão periódica baseia-se na revisão fixa do estoque, determinado pelo cálculo de tempo entre as revisões (TR), onde a quantidade de ressuprimento é obtida com base no estoque máximo.

O cálculo do Tempo entre as Revisões (TR), descrito na Tabela 2, considera o lote econômico de compra (LEC); o T ano que representa a quantidade de dias do período estudado; e a demanda (D) que é representada pela soma do consumo mensal.

O estoque de segurança (ES) para a metodologia de revisão periódica apresentando na tabela 2, também considera o intervalo de pedidos (IP) que corresponde ao tempo entre as revisões (TR).

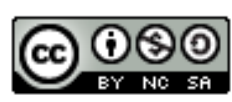


Para o cálculo do estoque máximo, conforme Tabela 2, considera-se o estoque de segurança (ES) e o lote econômico de compra (LEC).

\section{RESULTADOS E DISCUSSÃO}

\subsection{Simulação do modelo de revisão contínua}

Os resultados obtidos na Tabela 8 para os cálculos realizados do tempo entre estoque de segurança, ponto de pedido e lote econômico de compra seguem de acordo com as fórmulas apresentadas na Tabela 2.

Tabela 8. Sistema de Revisão Contínua

\begin{tabular}{lr} 
Fórmula & Resultado \\
\hline Estoque de Segurança & 644,61 \\
\hline Ponto do Pedido (PP) & 806,51 \\
\hline Lote Economico de Compra (LEC) & 1240,53 \\
\hline
\end{tabular}

Fonte: Autores (2021).

4.1.1 Resultados dos indicadores de desempenho modelo de revisão contínua

A tabela 9 apresenta os seguintes dados mensais: estoque médio mensal, a média do estoque médio, o número de pedidos realizados e o somatório de faltas do produto no estoque ocorridas. A metodologia para o cálculo desses itens segue a mesma metodologia utilizada para modelo atual, bem como para os cálculos dos indicadores giro de estoque e cobertura de estoque.

Tabela 9. Resumo do modelo de gestão de revisão contínua

\begin{tabular}{lcccccc} 
Meses & jan/21 & fev/21 & mar/21 & abr/21 & mai/21 & jun/21 \\
\hline Estoque médio & 2952,28 & 2415,00 & 2277,81 & 2121,14 & 1157,24 & 867,98 \\
\hline Média do Estoque médio & \multicolumn{7}{c}{1965,24} \\
\hline $\mathrm{n}^{0}$ de Pedidos & 0 & 0 & 0 & 0 & 1 & 1 \\
\hline$\Sigma$ Faltas & \multicolumn{7}{c}{573,46} \\
\hline Giro de Estoque & \multicolumn{7}{c}{2,97} \\
\hline Cobertura de Estoque & & 60,69 & & \\
\hline
\end{tabular}

Fonte: Autores (2021).

O cálculo do custo total mensal segue também segue a mesma metodologia utilizada no modelo atual, onde apenas o valor do custo de manutenção varia conforme o estoque médio. A tabela 10 apresenta a consolidação das informações referente ao custo de estoque.

Tabela 10. Custo Total do modelo de gestão de revisão contínua

\begin{tabular}{|c|c|c|c|c|c|c|c|c|c|c|c|c|}
\hline Meses & \multicolumn{2}{|c|}{ jan/21 } & \multicolumn{2}{|c|}{ fev/21 } & \multicolumn{2}{|c|}{$\mathrm{mar} / 21$} & \multicolumn{2}{|c|}{$a b r / 21$} & \multicolumn{2}{|c|}{$\mathrm{mai} / 21$} & \multicolumn{2}{|c|}{ jun/21 } \\
\hline Custo total de Pedido & $\mathrm{R} \$$ & - & $\mathrm{R} \$$ & - & $\mathrm{R} \$$ & - & $\mathrm{R} \$$ & - & $\mathrm{R} \$$ & 4,85 & $\mathrm{R} \$$ & 4,85 \\
\hline Número dos pedidos (NP) & & \multicolumn{2}{|c|}{0} & \multicolumn{2}{|c|}{0} & \multicolumn{2}{|c|}{0} & & \\
\hline Custo de Pedidos (CP) & $\mathrm{R} \$$ & 4,85 & $\mathrm{R} \$$ & 4,85 & $\mathrm{R} \$$ & 4,85 & $\mathrm{R} \$$ & 4,85 & $\mathrm{R} \$$ & 4,85 & $\mathrm{R} \$$ & 4,85 \\
\hline
\end{tabular}

\begin{tabular}{|c|c|c|c|c|c|c|c|c|c|c|c|}
\hline Custo total de Manutenção & $\mathrm{R} \$ 35.493,08$ & $\mathrm{R} \$$ & $29.033,73$ & $\mathrm{R} \$$ & $27.384,34$ & $\mathrm{R} \$$ & $25.500,86$ & $\mathrm{R} \$$ & $13.912,68$ & Rs & $10.435,12$ \\
\hline Estoque Médio (EM) & 2952,28 & & 2415,00 & & 2277,81 & & 2121,14 & & 1157,24 & & 867,98 \\
\hline Custo de Estocagem (CE) & 12,02 & $\mathrm{R} \$$ & 12,02 & $\mathrm{R} \$$ & 12,02 & $\mathrm{R} \$$ & 12,02 & $\mathrm{R} \$$ & 12,02 & $\mathbf{R}$ & 12,02 \\
\hline
\end{tabular}

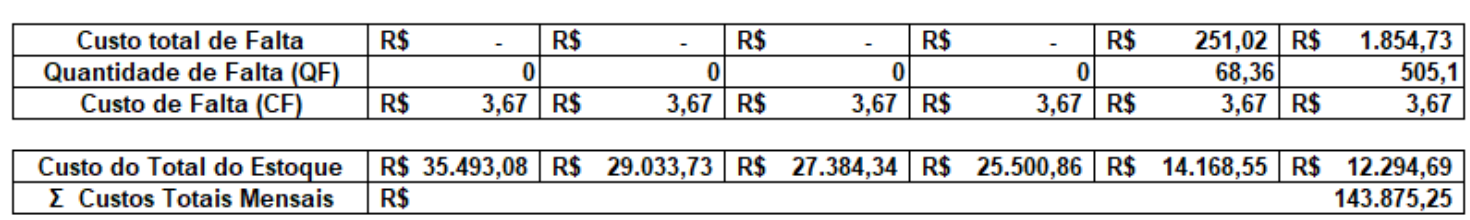

Fonte: Autores (2021).

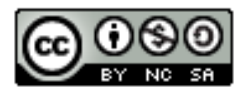


Citação (APA): Rodrigues, E. B., Rodrigues, M. E. B., Oliveira, M. C. F., de, Abreu, S. R., de, \& Oliveira, A. E., de. (2021). Melhoria no processo de gestão de estoque em uma confecção do ramo têxtil: Um estudo de caso. Brazilian Journal of Production Engineering, 7(5), 8292.

\subsection{Simulação do modelo de revisão periódico}

Os resultados obtidos na Tabela 11 para os cálculos realizados do tempo entre as revisões, estoque de segurança e estoque máximo seguem de acordo com as fórmulas apresentadas na Tabela 2.

Tabela 11. Sistema de Revisão Periódico

\begin{tabular}{lr} 
Formúla & Resultado \\
\hline Tempo ótimo entre as revisões (dias) & 38,31 \\
\hline Tempo ótimo entre as revisões (mês) & 1,26 \\
\hline Estoque de Segurança $(\mathrm{m})$ & 1885,71 \\
\hline Estoque Máximo $(\mathrm{m})$ & 3126,23 \\
\hline
\end{tabular}

Fonte: Autores (2021).

\subsubsection{Resultados dos indicadores de desempenho modelo revisão periódica.}

A Tabela 12 apresenta os seguintes dados mensais: estoque médio mensal, a média do estoque médio, o número de pedidos realizados e o somatório de faltas do produto no estoque ocorridas. A metodologia para o cálculo desses itens segue a mesma metodologia utilizada no modelo atual, assim como para os cálculos dos indicadores giro de estoque e cobertura de estoque.

Tabela 12 - Resumo do modelo de gestão de revisão periódica.

\begin{tabular}{lcccccc} 
Meses & jan/21 & fev/21 & mar/21 & abr/21 & mai/21 & jun/21 \\
\hline Estoque médio & 2952,28 & 2846,82 & 3064,83 & 3067,33 & 1543,20 & 1909,75 \\
\hline Média do Estoque médio & \multicolumn{7}{c}{2564,04} \\
\hline$N^{\circ}$ de Pedidos & 0 & 1 & 1 & 0 & 0 & 1 \\
\hline$\Sigma$ Faltas & \multicolumn{7}{c}{0} \\
\hline Giro de Estoque & \multicolumn{7}{c}{2,27} \\
\hline Cobertura de Estoque & \multicolumn{7}{c}{79,19} \\
\hline
\end{tabular}

Fonte: Autores (2021).

O cálculo do custo total mensal segue também segue a mesma metodologia utilizada no modelo atual, onde apenas o valor do custo de manutenção varia conforme o estoque médio. A Tabela 13 apresenta a consolidação das informações referente ao custo de estoque.

Tabela 13. Custo Total do modelo de gestão periódica

\begin{tabular}{|c|c|c|c|c|c|c|c|c|c|c|c|}
\hline Meses & jan/21 & \multicolumn{2}{|c|}{ fev/21 } & \multicolumn{2}{|c|}{$\mathrm{mar} / 21$} & \multicolumn{2}{|c|}{$a b r / 21$} & \multicolumn{2}{|c|}{ mai/21 } & \multicolumn{2}{|c|}{ jun/21 } \\
\hline Custo total de Pedido & $\mathrm{R} \$$ & $\mathrm{R} \$$ & 4,85 & $\mathrm{R} \$$ & 4,85 & R\$ & - & $\mathrm{R} \$$ & - & $\mathrm{R \$}$ & 4,85 \\
\hline Número dos pedidos (NP) & 0 & & & & & & & & & & \\
\hline Custo de Pedidos (CP) & 4,85 & $\mathrm{R} \$$ & 4,85 & $\mathrm{R} \$$ & 4,85 & $\mathrm{R} \$$ & 4,85 & $\mathrm{R} \$$ & 4,85 & $\mathrm{RS}$ & 4,85 \\
\hline
\end{tabular}

\begin{tabular}{|c|c|c|c|c|c|c|c|c|c|c|}
\hline Cus & $2 \$ 46.307,54$ & $\mathrm{R}$ & 33 & RS & 2,92 & $\mathrm{R} \$$ & 12,14 & $\mathrm{RS}$ & $24.205,59$ & R\$ \\
\hline & 28 & & 82 & & 83 & &, 33 & & 3,20 & \\
\hline Custo de Estocagem (C & 15,69 & $\overline{R S}$ & 15,69 & $\mathrm{R}$ & 15,69 & $\mathrm{R \$}$ & 15,69 & R & 15,69 & 15,69 \\
\hline
\end{tabular}

\begin{tabular}{|c|c|c|c|c|c|c|c|c|c|c|c|c|}
\hline Custo total de Falta & $\mathrm{R} \$$ & - & $\mathrm{R} \$$ & - & $\mathrm{R} \$$ & - & $\mathrm{R} \$$ & - & $\mathrm{R} \$$ & - & $\mathrm{R} \$$ & - \\
\hline Quantidade de Falta (QF) & & 0 & & 0 & & 0 & & 0 & & 0 & & 0 \\
\hline Custo de Falta (CF) & R\$ & 3,67 & R\$ & 3,67 & R\$ & 3,67 & R\$ & 3,67 & $\mathrm{R} \$$ & 3,67 & R\$ & 3,67 \\
\hline
\end{tabular}

\begin{tabular}{|c|c|c|c|c|c|c|c|c|c|c|}
\hline Custo do Total do Estoque & $\mathrm{R} \$ 46.307,54$ & $\mathrm{R} \$$ & $44.658,18$ & $\mathrm{R} \$$ & $48.077,77$ & $\mathrm{R} \$$ & $48.112,14$ & $\mathrm{R} \$$ & $24.205,59$ & R\$ $29.959,93$ \\
\hline$\Sigma$ Custos Totais Mensais & $\mathrm{R} \$$ & & & & & & & & & $241.321,14$ \\
\hline
\end{tabular}

Fonte: Autores (2021).

Para determinar qual modelo de gestão de estoque é o mais adequado para a confecção, foi realizada a comparação de todos os indicadores estoque médio, giro de estoque, cobertura de

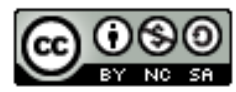


Citação (APA): Rodrigues, E. B., Rodrigues, M. E. B., Oliveira, M. C. F., de, Abreu, S. R., de, \& Oliveira, A. E., de. (2021). Melhoria no processo de gestão de estoque em uma confecção do ramo têxtil: Um estudo de caso. Brazilian Journal of Production Engineering, 7(5), 8292.

estoque e custo total de cada modelo de gestão de estoque. A tabela 14 apresenta um compilado dos resultados obtidos pelos indicadores em cada modelo de gestão de estoque.

Tabela 14. Compilado dos indicadores dos modelos de gestão de estoque Indicador Modelo Atual Modelo de Revisão Contínua Modelo de Revisão Períodica

\begin{tabular}{|c|c|c|c|}
\hline & & da & ão Períodica \\
\hline Média do Estoque Médio (m) & 4263,73 & 1965,24 & 2564,04 \\
\hline$\overline{\Sigma \text { Faltas (unid.) }}$ & 0 & 573,46 & 0 \\
\hline Giro de Estoque (vezes) & 1,37 & 2,97 & 2,27 \\
\hline Cobertura de Estoque (dias) & 131,68 & 60,69 & 79,19 \\
\hline$\overline{\Sigma \text { Custo Total do Estoque }}$ & $667.282,35$ & $143.875,25$ & $241.321,14$ \\
\hline
\end{tabular}

Fonte: Autores (2021).

Analisando o somatório do custo total dos itens estudados para o modelo de gestão atual utilizado pela empresa tem-se o valor de $\mathrm{R} \$ 667.282,35$, e para o modelo de revisão contínua é de $\mathrm{R} \$ 143.875,25$, representando uma economia de 78,44\% do modelo de revisão contínua para o modelo atual no custo do estoque. Realizando a mesma análise para o modelo de revisão periódica, tem-se o somatório do custo total do estoque de $\mathrm{R} \$ 241.321,14$, o que representa uma economia $63,84 \%$ sobre o modelo atual de estoque. Observa-se que a falta de um modelo de gestão de estoque agrava o custo total do estoque. Uma vez que ambos os modelos de gestão apresentado como alternativa saiu-se de forma positiva sobre o modelo de ressuprimento que a empresa atualmente usa. Também é possível observar que o custo total do estoque está diretamente relacionado a média do estoque, onde o modelo atual possui a maior média de 4263,73 metros e o modelo que se demonstrou mais econômico tem a menor média de 1965,24 metros. Sendo assim, procurar manter a média de estoque menor, reduz o custo total do estoque.

\section{CONCLUSÃO}

O estudo realizado neste trabalho apresenta resultados e análises acerca das simulações realizadas no estoque de uma empresa do ramo têxtil, a fim de identificar qual modelo de gestão de estoque é o mais ideal para ressuprimento do estoque. A opção mais vantajosa conforme mostrado na tabela 14, é a utilização do modelo de revisão contínua, por resultar em uma economia de 78,44\% no custo total do estoque, porém este mesmo modelo apresentou faltas de matéria prima na simulação, resultando uma falta total 573,46 metros. Este fator foi determinante ao apresentar os resultados dos modelos propostos para o atual gestor da empresa, onde por mais que o custo de falta esteja considerado no custo total, e mesmo assim ter apresentado uma maior economia, para o gestor as faltas representariam atrasos produção, que por sua vez representam atrasos nas entregas dos produtos aos clientes.

Desta maneira o modelo que mais demonstrou-se ideal para a gestão de estoque da empresa estudada foi o modelo de revisão periódica, já que não houve faltas na simulação que representaria inatividade operacional na produção.

\section{REFERÊNCIAS BIBLIOGRÁFICAS}

Associação Brasileira da Indústria Têxtil e de Confecção - ABIT

Bowersox, D. J.; Closs, D. J. (2001). Logística empresarial: o processo de integração da cadeia de suprimentos. São Paulo: Atlas.

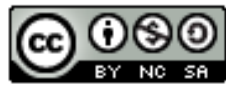


Citação (APA): Rodrigues, E. B., Rodrigues, M. E. B., Oliveira, M. C. F., de, Abreu, S. R., de, \& Oliveira, A. E., de. (2021). Melhoria no processo de gestão de estoque em uma confecção do ramo têxtil: Um estudo de caso. Brazilian Journal of Production Engineering, 7(5), 8292.

Ching, H. Y. (2010). Gestão de estoques na cadeia de logística integrada: supply chain. 4. ed. São Paulo: Atlas.

Chopra, S., \& Meindl, P. (2016). Gestão da cadeia de suprimentos: estratégia, planejamento e operações. 6. ed. São Paulo: Person.

Corrêa, H. L., \& Corrêa, C.A. (2012). Administração da produção e operações: Manufatura e

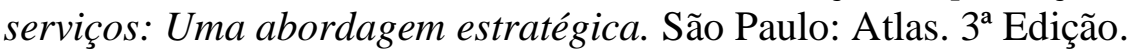

Dias, M. A. P. (2010). Administração de Materiais: Princípios, Conceitos e Gestão. 6. ed. São Paulo: Atlas.

Gasnier, D. G. (2002). A dinâmica dos estoques: guia prático para planejamento, gestão de materiais e logística. São Paulo: IMAM.

Martins, P. G., \& Alt, P. R. C. (2006). Administração de materiais e recursos patrimoniais. 2. ed. São Paulo: Saraiva.

Martins, P. G., \& Campos A., P. R. C. (2009). Administração de Materiais e Recursos Patrimoniais. São Paulo:Saraiva.

Moreira, D. (2012). Administração da Produção e Operações. $2^{\mathrm{a}}$ Edição. São Paulo: Cengage Learning.

Paoleschi, B. (2014). Estoques e armazenagem. $1^{\text {a }}$ ed. Érica. São Paulo.

Parente, J. (2009). Varejo no Brasil: gestão e estratégia. São Paulo Atlas.

Peinado, J., \& Graeml, A. (2007). Administração da Produção: Operações Industriais e Serviços. Curitiba: UnicenP.

Pozo, H. (2007). Administração de recursos materiais e patrimoniais: uma abordagem logística. 4. ed. São Paulo: Atlas.

Slack, N., Jones, A, \& Johnston, R. (2018). Administração da Produção. 8 ed. São Paulo: Atlas.

Soto, D. L., Angel-Bello, F., Yacout, S., \& Alvarez, A. (2017). A multistart algorithm to design a multiclass classifier for a multicriteria $\mathrm{ABC}$ inventory classification problem. Expert Systems With Applications, 81, 12-21.

Tubino, D. F. (2000). Manual De Planejamento E Controle De Produção. São Paulo: Editora Atlas S.A, 2 Edição.

Tubino, D. F. (2007). Planejamento e controle da produção: teoria e prática. 2 ed. São Paulo: Editora Atlas.

Wanke, P. Gestão de Estoque na Cadeia de Suprimento. 3.ed. São Paulo: Atlas, 2011. 